\title{
Mobility Issue on Octagonal Structured ZigBee Network Using Riverbed
}

\author{
Nazrul Islam¹, Md. Jaminul Haque Biddut', Md. Faizul Huq Arif', \\ Mohammad Motiur Rahman², Md. Syfur Rahman ${ }^{3}$ \\ ${ }^{1}$ Department of Information and Communication Technology (ICT), Mawlana Bhashani Science and Technology \\ University, Santosh, Tangail, Bangladesh \\ ${ }^{2}$ Department of Computer Science and Engineering (CSE), Mawlana Bhashani Science and Technology \\ University, Santosh, Tangail, Bangladesh \\ ${ }^{3}$ Education Management Information System (EMIS) Cell, Directorate of Secondary and Higher Education, \\ Ministry of Education, Dhaka, Bangladesh \\ Email: nazrul.islam@mbstu.ac.bd, biddutict@gmail.com, arifict27@gmail.com, \\ mm73rahman@gmail.com, syfur022@gmail.com
}

Received 2 January 2016; accepted 6 March 2016; published 29 March 2016

Copyright (C) 2016 by authors and Scientific Research Publishing Inc.

This work is licensed under the Creative Commons Attribution International License (CC BY).

http://creativecommons.org/licenses/by/4.0/

(c) (i) Open Access

\begin{abstract}
Wireless Sensor Network (WSN) is a special type of communication medium through distributed sensor nodes. Popular wireless sensor nodes like ZigBee have splendid interoperability after IEEE 802.15.4 standardization in the domain of wireless personal area network (WPAN). ZigBee has another great feature mobility that makes the ZigBee network more versatile. The mobility feature of ZigBee mobile nodes has a greater impact on network performance than fixed nodes. This impact sometimes turns into more severe because of network structure and mobility model. This study mainly focuses on the performance analysis of the ZigBee mobile node under Random and Octagonal mobility management model with the Tree routing method. The Riverbed academic modeler is used to design, implement and simulate the ZigBee network under certain conditions. This study also presents a competitive performance analysis based on ZigBee mobile nodes transmitter and receiver characteristics under the observation of the mobility model. This indicates that Octagonal mobility model exhibits better performance than the Random mobility model. This study will constitute a new way for further designing and planning a reliable and efficient ZigBee network.
\end{abstract}

\section{Keywords}

WSN, ZigBee Network, Mobility, Octagonal Structure, Riverbed 


\section{Introduction}

Wireless Sensor Networks (WSNs) are highly integrated technologies comprising of sensors, microcontrollers and wireless networking capabilities which autonomously collect data and send data through a sink. Nowadays, it is one of the most emerging research fields in the world. WSNs have been in development for many years to benefit better performance and widespread applications. Static sink based wireless sensor networks were deployed in the past but mobile sink has changed the direction in the research field. Mobility in wireless sensor network has drawn much attention to the researchers in the recent years.

In that direction, mobility in ZigBee/IEEE 802.15.4 as a WSN has turned to open a vast field for researchers. The ZigBee devices facilitate widespread applications in home and building automation with low cost, low data rate and reliable communications [1] [2]. The ZigBee WSNs provide security, pervasive computing, monitoring, traffic control, vehicular safety access control and location based information pushing and so on [3]-[5]. Recently, several studies have been done on different performance analysis and enhancement of management approach for ZigBee mobile nodes [6] [7]. The study [6] describes ZigBee based wireless sensor networks mobile sink implementation in terms of various mobility strategies. Moreover, performance and fairness improvement technique are also analyzed in the thesis [7]. The article [8] proposed a scalable approach to avoid traffic overhead caused by routing protocol and the performance of ZigBee mobile nodes has also been studied. Ddin et al. presented improved Quality of Service (QoS) with ZigBee mobile sink [9]. The study [10] presented an enhanced approach for mobility management of end devices in ZigBee cluster tree network. Enhancing approach mainly occurs in transceiver of ZigBee devices. Like fixed nodes, each ZigBee mobile node has also transceiver for data sending and receiving from other devices. The execution of requests in the transceiver of ZigBee mobile node has enormously depended on its mobility model. The convenient mobility model supports to increase transceiver performance as well as mobile device performance. That services to develop ZigBee networks mobility feature.

Previously, mobility models only studied for small range ZigBee network [11]. After getting popularity of ZigBee's mobility feature, a convenient and reliable mobility model is now demanded for large scaled ZigBee network. To the best of our knowledge, mobility model for large scale ZigBee network has not designed until now. Moreover, Zigbee mobile node's performance based on its mobility model alongside with mobile node's transmitting and receiving capability has not studied yet. Therefore, further study is needed on ZigBee's sink mobility.

This study presents random and a unique octagonal structured ZigBee network to evaluate ZigBee mobile sink's mobility feature. The purpose of this study is to investigate and compare the lower level performance of the node's transmitter and receiver (on queue size, end to end delay, and bit error rate, signal to noise ratio and throughput of the nodes etc.) between these two schemes for mobility of sink. After analyzing the results, it has revealed that the octagonal mobility model based network shows better performance.

This paper is organized as follows: Section 2 presents the research methodology. Section 3 discusses the modeling and simulation of the network. Section 4 analyzes the result for mobility behavior and discusses the mobile nodes performance. Moreover, simulation results are analyzed in Section 5. Finally, it concludes the study with future work in Section 6.

\section{Research Methodology}

A precise literature review on the mobility issue of wireless sensor network has been served. Based on the literature review, mobility issues in ZigBee network has been selected for further study to develop ZigBee mobile node's performance. After the deep study, an enhanced way has been discovered. Recently, Biddut et al. in [12] presented a simulation based large-scale ZigBee's network performance analysis through geometric structure. The study shows that geometrical structure based network performance is better. From this study, a geometrical structure based network design criteria has adopted to increase ZigBee mobile node's transmitter and receiver sensitivity in the Octagonal configuration model than randomly placed sink, routers or other end devices. In the Octagonal configuration model, routers are fixed at vertices, end devices are oriented center at routers and mobile sink traverse through outer periphery of octagon structure. To implement such network model, an efficient simulator needed.

A deep study suggests many simulation tools such as Network Simulator-2 [13], Network Simulator-3 (NS-3) [14], OMNET++ [15], Riverbed [16] etc. support ZigBee network development features. In this study, the design, implementation and simulation of ZigBee network is carried out in a popularly Riverbed simulator for its 
wide range of acceptance. Riverbed academic modeler supports modeling of communication networks and distributed system.

After simulation, the different ZigBee protocol layers provide different types data. Simulated data is collected properly and carefully. Appropriate graphs are plotted with the simulated data. The graphs present the ZigBee mobile node's (transmitter and receiver) comparative performance analysis. The analysis is carried out with respect to the random and the octagonal configuration mobility model of ZigBee network.

\section{Simulation and Modeling}

In this section, the main focus is to design and discuss mobility issue of ZigBee network based on two different Random and Octagonal configuration model. In this simulation study, IEEE 802.15.4/ZigBee node cluster tree network has considered and figured out the performance of the network when nodes are moving.

\subsection{Model Design and Implementation}

The ZigBee network is design based on Tree routing scheme for both of the network model. Tree routing is an efficient routing method for ZigBee's application traffic distribution [2] [17]. Where the nodes move randomly within the topology. Each node transmits data frame of 1024 bytes which are routed through the network to the Coordinator (Sink). The Coordinator moves throughout the network field. To setup a consistent network field within which consists of the end devices that sense the data and transmit via the routers or directly to the Coordinator (Sink). The network assumptions are as follows:

The network field is a square shaped area. The routers arrangement in either one of the two configurationsrandom and octagon. The Coordinator can be static (at the center of the network field) or mobile. The mobile Coordinator follows the outer periphery as a path of Octagonal and Random model. Moreover, the external interference is considered zero.

\subsection{Mobility Model}

Mobility means the movement of sensor nodes through the entire network field. In real time communication, mobility is one of the characteristics of WSNs. Three different types of mobility have been suggested: random, predictable or fixed and controlled in previous works [6] [10].

Before discussing in detail, it is worth noting that the privileges of particular sink mobility depend on the application model of underlying WSN-which can be either continuous or event based. In continuous model, all sensor nodes transmit data to the Coordinator (Sink) (e.g. Environment monitoring application). On the other hand, only a small subset of nodes sends data to sink at different instances of time (e.g. Target tracking application). This paper states the analysis of the performance of mobile ZigBee nodes on two different mobility strategies-Random mobility model and Octagonal mobility model. Random model is an arbitrary distribution of sensor nodes while Octagonal model is more fixed and predictable. Besides, Octagon is a popular shape for designing a large building. Several Octagonal shapes are comprised of complex, gigantic building. Moreover, in geometric context, Octagonal shape based building design is an optimal solution for planning complex building design than the other shapes like a hexagon, triangular, circular and so on [18]. This study mainly compares the performance of the Octagonal model with Random model. The overview of two different models is presented in Figure 1. In this figure, the white line represents Coordinator trajectory. Coordinator moves through this trajectory in both the Random and Octagonal mobility model. In addition, circular points are the ZigBee nodes.

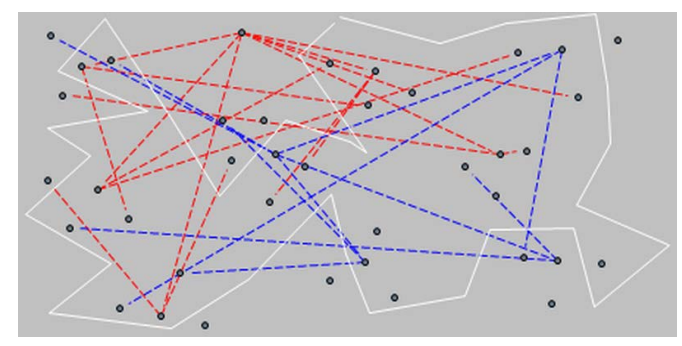

(a)

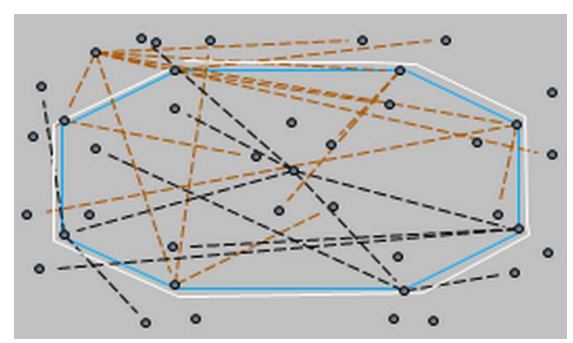

(b)

Figure 1. Random and octagonal mobility model. (a) Random; (b) Octagonal. 


\subsubsection{Random Configuration Mobility Model}

In this configuration, routers and end devices are distributed throughout the network field randomly. The destination point is also in a random manner. In this model, the trajectory of sink movement in random manner. Random mobility is suitable for continuous monitoring in application perspective.

\subsubsection{Octagonal Configuration Mobility Model}

In this configuration model, routers are arranged so as to cover the entire network field and forming octagon. The vertices of the octagon represent the routers. The routers are within radio range of two adjacent routers. In this model, the trajectory of sink movement is fixed path. The mobile sink is deterministic. During the entire simulation period, it moves outside the network field on the boundary forming an octagon.

\section{Model Simulation}

The simulation was set in two different scenarios. Scenario_1 is setup in the Random configuration mobility model. The random movement of mobile node has been implemented using the Riverbed simulator with its random mobility feature. Figure 2 shows nodes are scattered randomly. Coordinator runs through the random white trajectory as depicted in this figure. The ZigBee routers and other nodes have placed randomly in the network surface. On the other hand, Scenario_2 is setup in the Octagonal configuration mobility model. Figure 3 shows the position of the router which presents the vertices of an octagonal shape. The Coordinator also moves through the octagonal trajectory. The simulation is done with two configurations in a range of $500 \mathrm{~m}^{2}$ within $2.45 \mathrm{GHz}$ frequency bands.

The overall configuration comprises of 39 ZigBee nodes, in which-8 routers, 30 end devices and 1 coordinator. Though, large scale ZigBee network is not yet established, a ZigBee Coordinator can support up to 65,535 devices at a time [2]. From this motivation, an extensive simulation configured with 39 devices which can be an assumption for the effect of mobility models in large-scale perspective. Moreover, the network dimension also covers up to $500 \mathrm{~m}^{2}$ area. The mobile coordinator moves at a fixed speed of $1 \mathrm{~m} / \mathrm{s}$. In Scenario_1, the mobile sink moves randomly throughout the entire network field. On the other hand, in Scenario_2 the mobile sink moves in an octagonal periphery.

The two scenarios follow Tree routing method for its application traffic forwarding. The address allocation tree structure be same for moving nodes in this case also. Both the scenarios work for allocating device addressing as follows:

$$
\begin{gathered}
C_{\text {Skip }_{d}}=0 \text {, when } R_{m}=0 \\
C_{\text {Skip }_{d}}=\frac{1-C_{m}-R_{m}-C_{m} R_{m}^{L_{m}-d-1}}{1-R_{m}} \text {, when } R_{m}>1 \\
C_{\text {Skip }_{d}}=1+C_{m}\left(L_{m}-d-1\right) \text {, when } R_{m} \neq 1
\end{gathered}
$$

where, $C_{m}$ presents the largest number of child which can associate with a router or Coordinator. $R_{m}$ represents the number of child nodes which can be a router. $L_{m}$ presents the most depth of the network with respect to different nodes have the constant $C_{m}$ and $R_{m}$. Every parent is provided with a finite set of address space which usually assigned for the network address to its children. $C_{\text {Skip }_{d}}$ acts as the size of the address sub-block distributed by each parent at depth $d$. When $C_{\text {Skip }}$ of a node is 0 , it is unable to distribute address for the children. If $C_{\text {Skip }_{d}}$ is greater than 0 then the node can accept other nodes as its child and distribute network address for its child nodes.

Moreover, network addresses must be assigned to $n$-th routers child and 1-st end device child at depth $d+1$ in a sequential manner respectively as followings:

$$
\begin{gathered}
A_{d+1, r n}=A_{\text {Parent }}+C_{\text {Skip }_{d}}(n-1)+1 \\
A_{d+1, \text { el }}=A_{\text {Parent }}+C_{\text {Skip }_{d}} R_{m}+l
\end{gathered}
$$

where, $1 \leq l \leq R_{m}$ and $A_{\text {Parent }}$ represents the address of the parent. When $1 \leq l \leq\left(C_{m}-R_{m}\right)$ [12] [19]. 


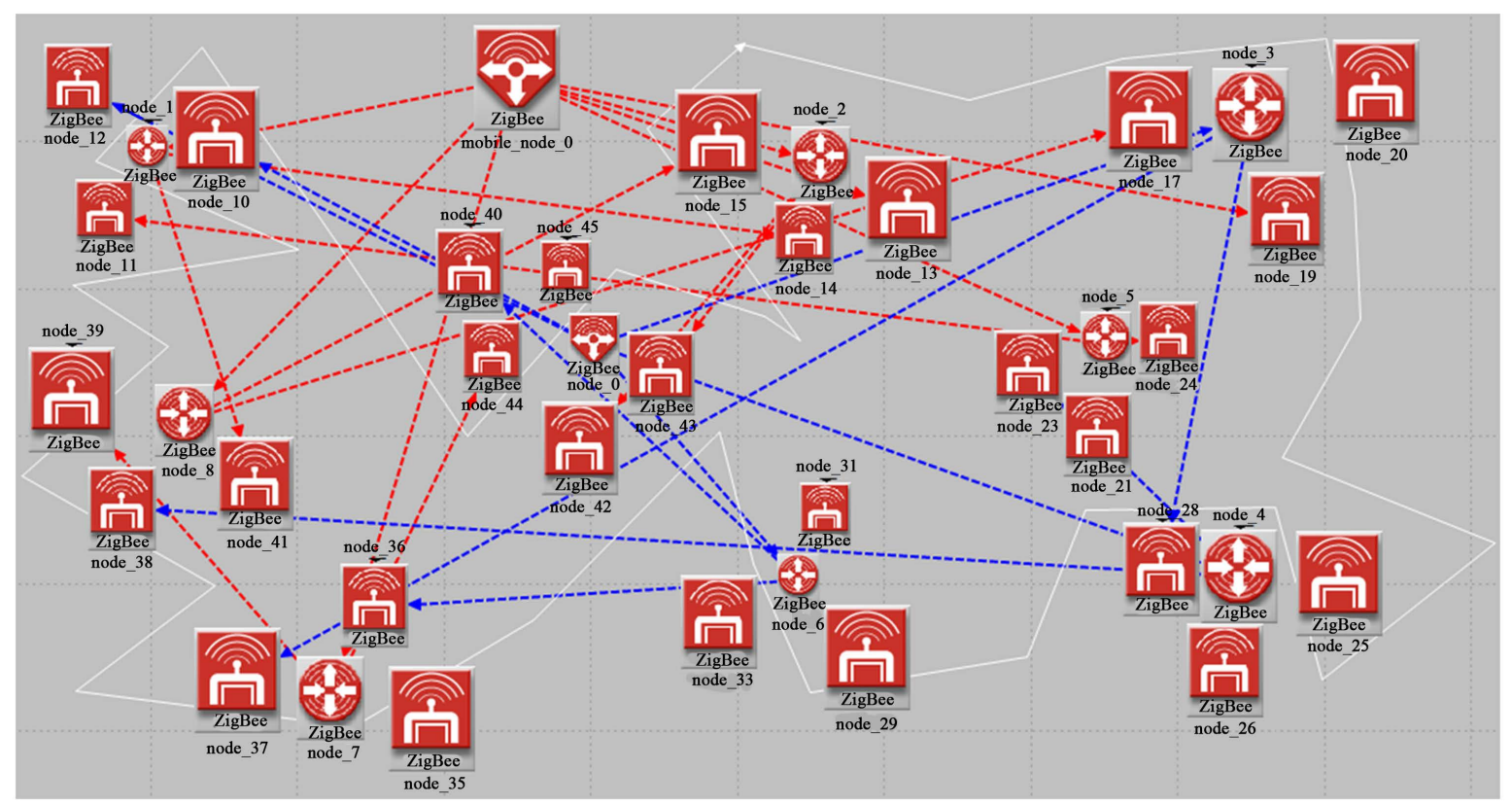

Figure 2. Random configuration mobility model (Scenario_1).

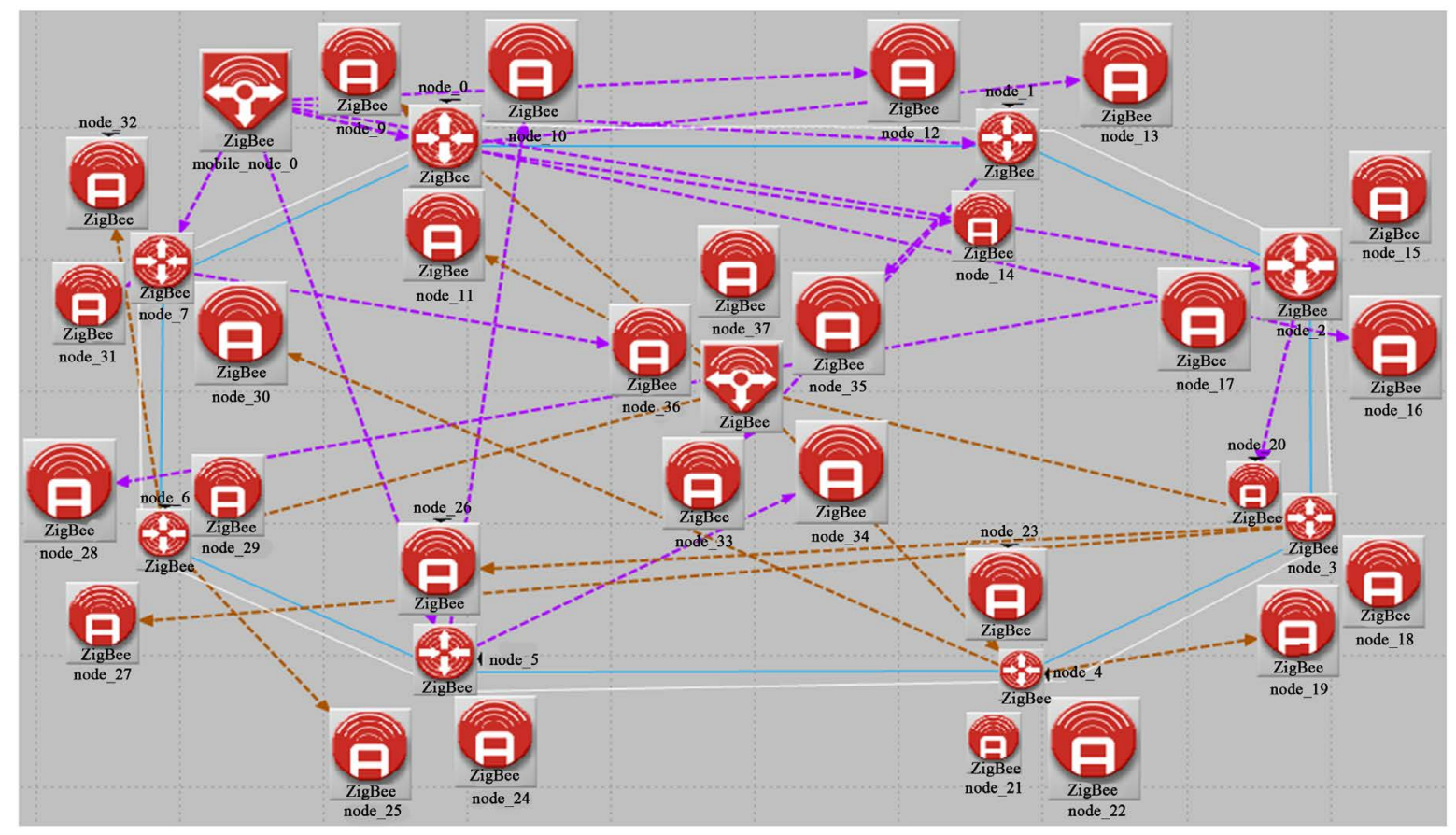

Figure 3. Octagonal configuration mobility model (Scenario_2).

The overall simulation time for both configurations is 600 sec. In this simulation, some key parameters including ACK status, data rate, packet size, retransmission attempts, packet destination, mobility type, moving speed, traffic destination etc. are kept constant as defined in Table 1.

\section{Result Analysis}

This section analyzes lower level data on ZigBee mobile node in details. The lower level data are the core level data from any ZigBee device. It does not mean Application, MAC or Network layer data. It is the core feature of 
Table 1. Simulation parameters overview.

\begin{tabular}{cc}
\hline Parameters & Descriptions \\
ACK status & Enabled \\
No. of retransmissions & $5.45 \mathrm{GHz}$ \\
Frequency band & Auto calculate \\
Data rate & Constant (1024) \\
Packet size & Constant (1.0) \\
Packet interval time & $1 \mathrm{~m} / \mathrm{s}$ \\
Moving speed & Random and fixed \\
Mobility type & 0 \\
Pause probability & Coordinator \\
Traffic destination & 39 \\
No. of nodes (overall) & 1 \\
No. of coordinator & 8 \\
No. of routers & 29 \\
No. of end devices & $500 \mathrm{~m} \times 500 \mathrm{~m}$ \\
Network dimenson & 600 sec \\
Simulation time & \\
\hline
\end{tabular}

any ZigBee device for communication from device to device. The data are collected from extensive simulation on the basis of ZigBee mobile node's transmitter and receiver. The graphs illustrate, several characteristics of the mobile node's transceiver on its mobility issue for Octagonal and Random ZigBee configuration model.

\subsection{Wireless Transmitter (TX)}

In ZigBee, a transmitter is usually part of a communication system which uses electromagnetic waves (radio waves) to transport information over a distance which embedded in all ZigBee devices. Source end device collects data from its sensing capability and prepares it for transmission. The transmitter is responsible to send data from source to destination.

\subsubsection{Queue Size}

Queue size represents the total number of packets outstanding in the wireless transmitter (TX) queue. That indicates, the number of a sequence of request waiting to execute in transmitter (TX) queue. In the Random model, transmitter collects more data packets than the Octagonal model for its random traversing. All the data packets stay in transmitter queue. Figure 4, shows average queue size for Random model is approximately 1.5 times larger than Octagonal model.

Figure 4 also shows that the average packet in random configuration sink transmitter (TX) queue is more than Octagonal configuration sink queue. It is almost 2 times larger queue for Random model.

\subsubsection{Queuing Delay}

Queuing delay represents the delay that packets from the end nodes incurs at the wireless transmitter queue until it can be executed or transmitted. It is one of the vital component of overall network delay. More data packets in queue increase more delay. Figure 5, illustrates the average queuing delay in Random model is $13 \%$ higher than Octagonal model.

As data packets wait in queue for next execution, a delay occurs for waiting. In Random configuration, sink gets more data packets than Octagonal configuration sink. Due to getting more data packets described earlier, average waiting time in queue for total data packets is higher for the Random configuration. 
Transmitter Queue Size

_Geometric_Structured_Zigbee_Network-Octagonal_Config_Mobility-DES-1: Office Network.mobile_node_0.wireless_tx.channel [0].radio transmitter.queue size (packets)

_Geometric_Structured_Zigbee_Network-Random_Config_Mobility-DES-1: Office Network.mobile_node_0.wireless_tx.channel [0].radio transmitter.queue size (packets)

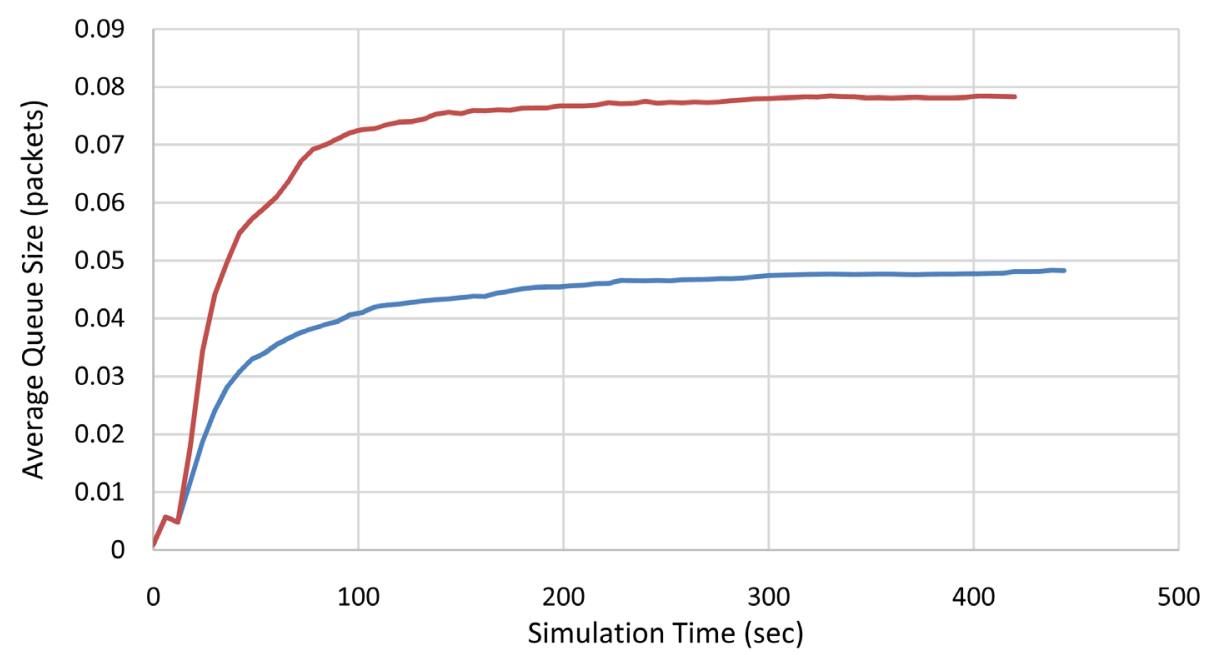

Figure 4. Average queue size (packets) for octagonal and random model.

Transmitter Queuing Delay

_-Geometric_Structured_Zigbee_Network-Octagonal_Config_Mobility-DES-1: Office

Network.mobile_node_0.wireless_tx.channel [0].radio transmitter.queuing delay (sec)

_Geometric_Structured_Zigbee_Network-Random_Config_Mobility-DES-1: Office Network.mobile_node_0.wireless_tx.channel [0].radio transmitter.queuing delay (sec)

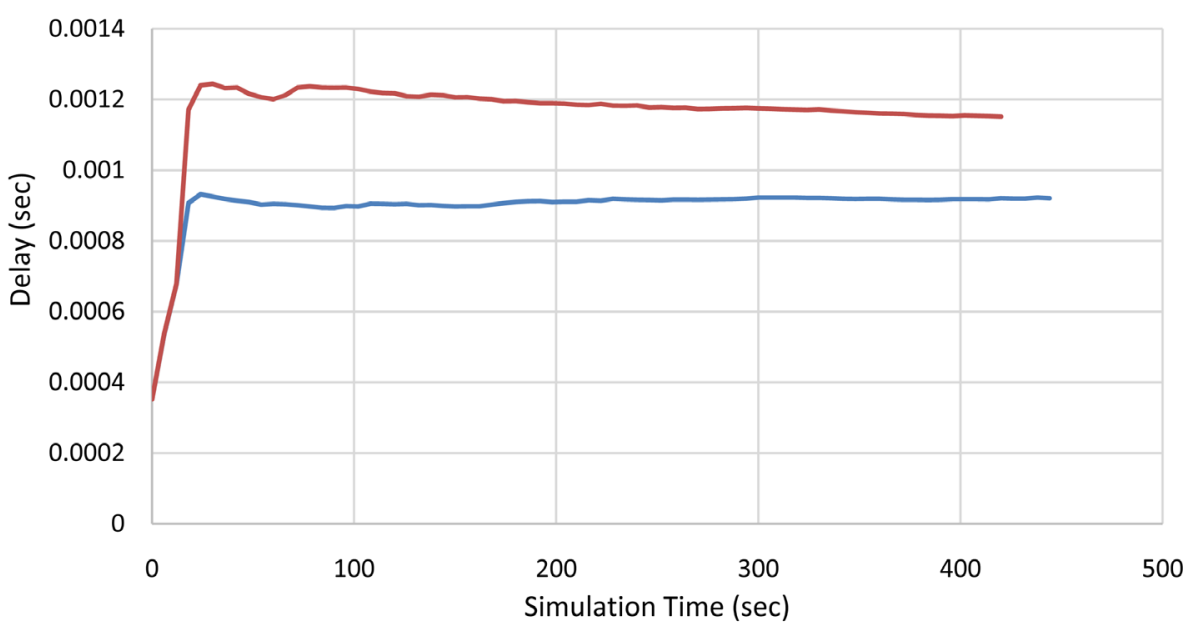

Figure 5. Average queuing delay (sec) for octagonal and random model.

\subsubsection{Throughput}

The mobile sink randomly traverse in the whole network. Thus, it collects more data packets to transfer which increases transmitter's throughput than the fixed octagonal traverse path model. Figure 6, describes the average throughput of Random configuration is almost 28\% higher than Octagonal configuration model.

The Random configuration model has more data packets availability than the Octagonal configuration model. The above discussion shows that Random configuration model has the larger queue size, higher queuing delay and throughput than Octagonal configuration. 


\section{Transmitter Throughput}
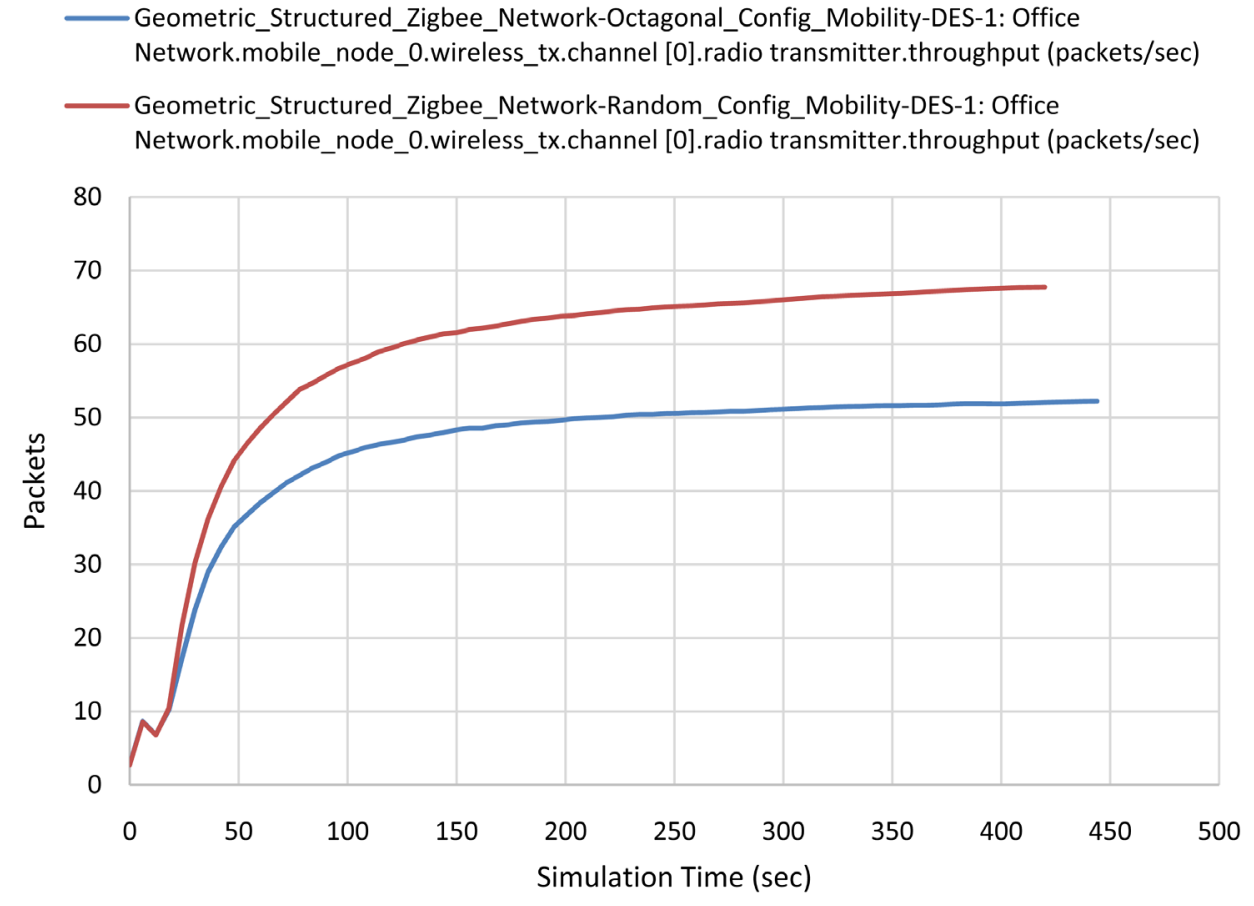

Figure 6. Average transmitter throughput (bits/sec) for octagonal and random model.

\subsection{Wireless Receiver (RX)}

In ZigBee, a receiver is an electronic device that receives radio waves and converts the information carried by them to a usable form. The receiver uses electronic filters to split up the desired radio frequency signal from all the other signals to recover the desired information through demodulation. After successfully receiving data receiver sends its processing unit for further execution.

\subsubsection{Bit Error Rate}

In digital transmission, the number of bit errors per unit time is the number of receiving bits of a data stream over a communication channel that have been altered due to noise, interference, distortion or bit synchronization errors.

Figure 7, shows the bit error rate in complete communication is about $21 \%$ lower and about $30 \%$ lower per packet for Octagonal configuration than Random configuration model.

Sink in the Random configuration model has more data packets to send. The more data packets which are affected by noise, synchronization error, interference etc. Thus, bit error rate per packet and whole communication medium are higher for the Random configuration model than the Octagonal configuration model.

\subsubsection{Packet Loss Ratio}

Packet loss occurs when one or more packets of data travelling across a ZigBee network fail to reach their destination. In the Octagonal configuration, routers and other end devices are fixed in a deterministic range and also mobile node has fixed traverse path. For this reason, regular communication, packet loss ratio is $22 \%$ higher in Random configuration model as described in Figure 8.

Sink in the Octagonal configuration model, traverse through the fixed octagonal periphery. Routers are placed at a fixed distance. Moreover, the sink transmitter queue is not overloaded. On the other hand, routers, end devices are placed randomly in communication field. Sink also traverses randomly throughout the whole area and lack of coverage one end to the other end of the communication field. Thus, the communication between sources to destination is successfully occurring in the Octagonal configuration model. The packet loss probability also lower compare to Random configuration model. 


\section{Receiver Bit Error rate}

Geometric_Structured_Zigbee_Network-Octagonal_Config_Mobility-DES-1: Office Network.mobile_node_0.wireless_rx.channel [0].radio receiver.bit error rate

_Geometric_Structured_Zigbee_Network-Random_Config_Mobility-DES-1: Office Network.mobile_node_0.wireless_rx.channel [0].radio receiver.bit error rate

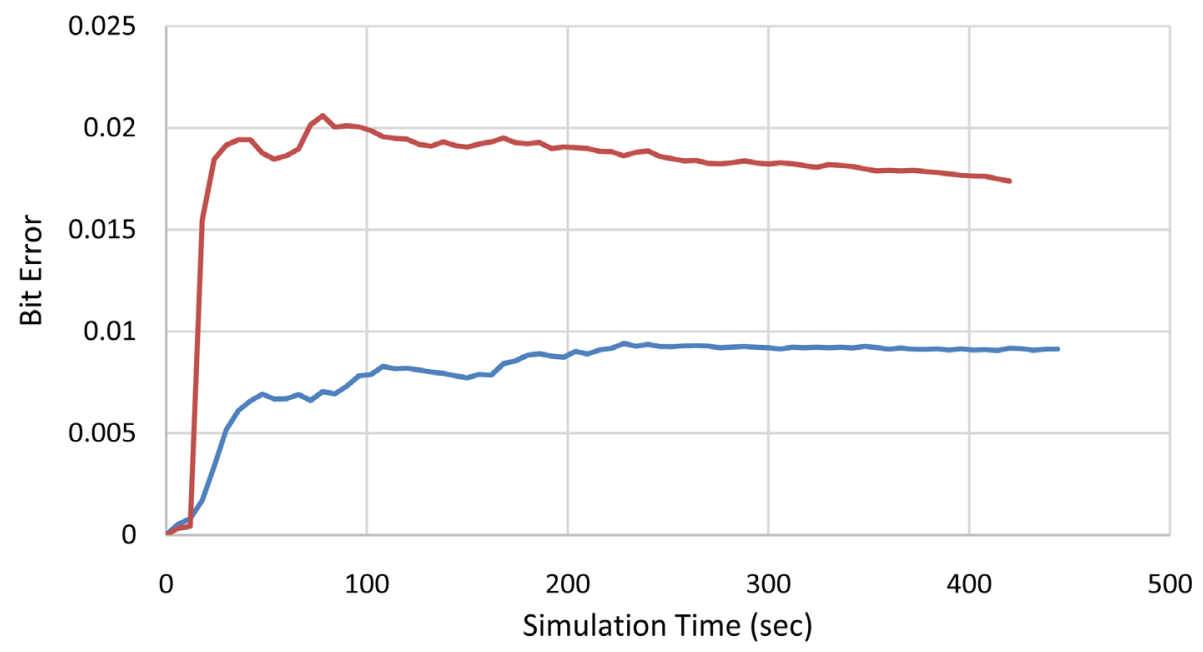

Figure 7. Average bit error rate for octagonal and random model.

Receiver Papcket Loss Ratio

—Geometric_Structured_Zigbee_Network-Octagonal_Config_Mobility-DES-1: Office Network.mobile_node_0.wireless_rx.channel [0].radio receiver.packet loss ratio

_Geometric_Structured_Zigbee_Network-Random_Config_Mobility-DES-1: Office Network.mobile_node_0.wireless_rx.channel [0].radio receiver.packet loss ratio

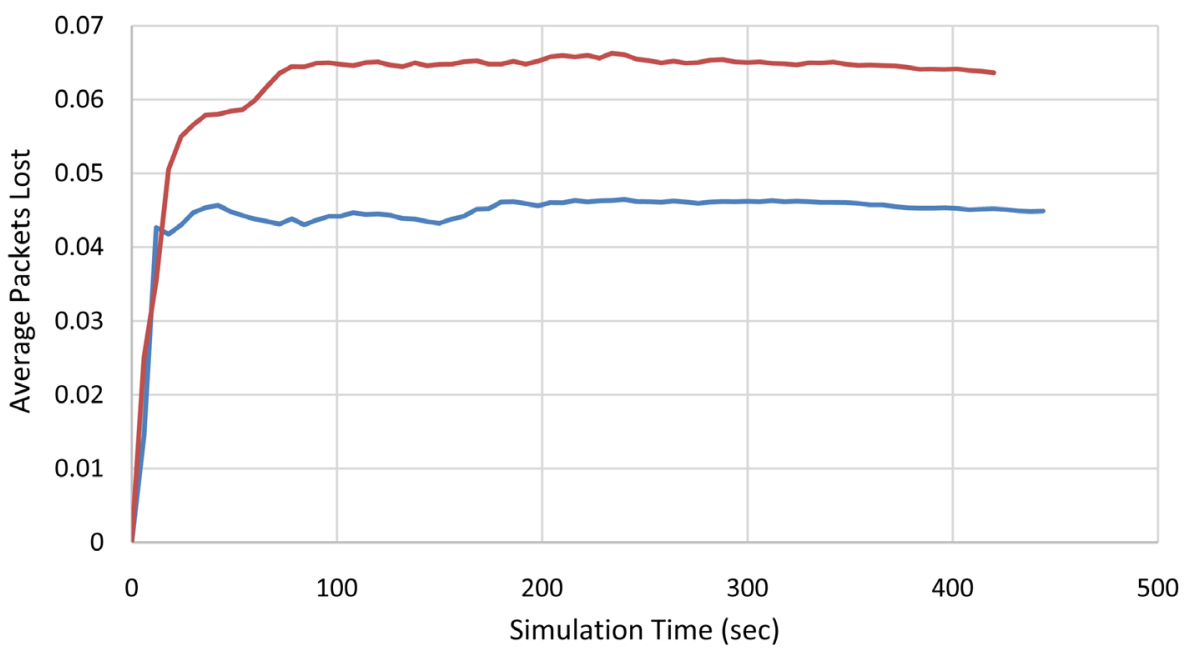

Figure 8. Average packet loss ratio for octagonal and random model.

\subsubsection{Signal to Noise Ratio}

Signal-to-Noise Ratio (SNR) is a measure used in ZigBee network that compares the level of a desired signal to the level of background noise. As mobile node traverse in octagonal periphery, its data delivery is in a sequential manner and less affected by external noise. On the other hand, external noise and interference affect mobile node more for random traversing. Figure 9, shows average SNR for Octagonal model is about $7 \%$ higher than Random model. 
Randomly placed nodes gets more affected by internal and external noise. These random nodes generate internal noise through its radio frequency. On the other hand, internal noise is much lower in the Octagonal configuration model.

\subsubsection{Throughput}

In Random model as it picks up more packets than Octagonal model, the average throughput for the receiver is 6\% higher for Random model as illustrated in Figure 10.

In Random model, mobile node gets more data packets and it sends more packets than Octagonal model. In this process, Random model faces larger queue size, lower signal to noise ratio, higher queuing delay, higher bit error rate, higher packet loss.

\section{Receiver Signal-to-Noise Ratio}

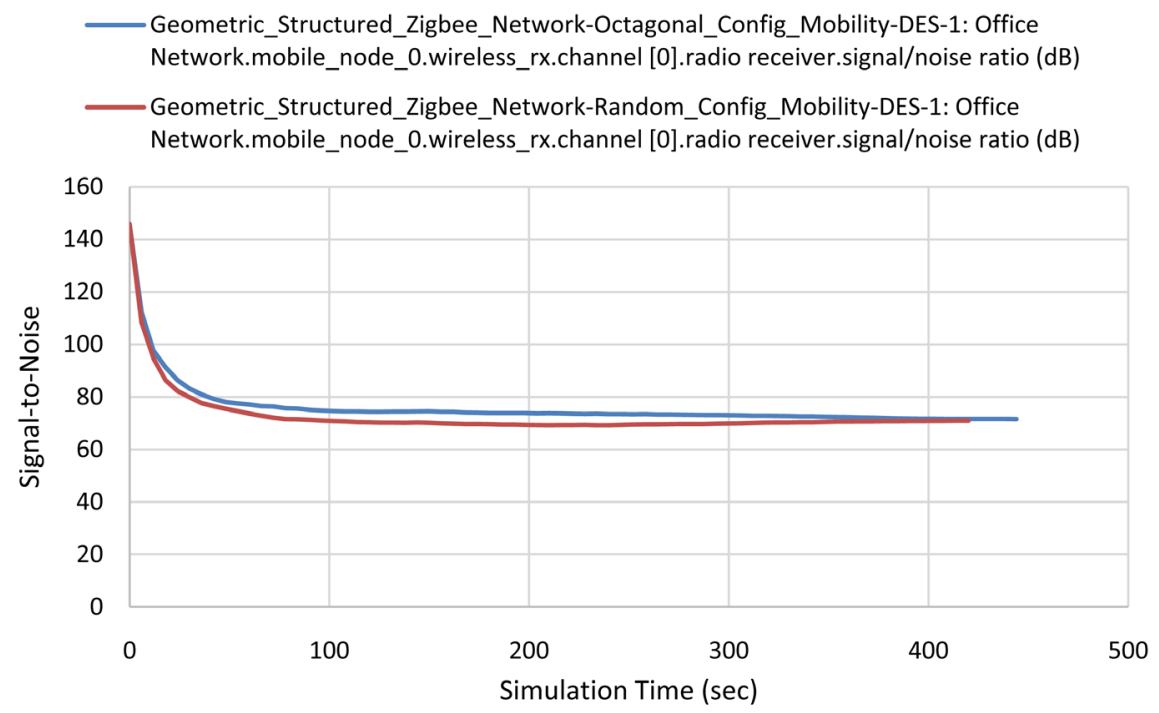

Figure 9. Average SNR for octagonal and random model.

Receiver Throughput

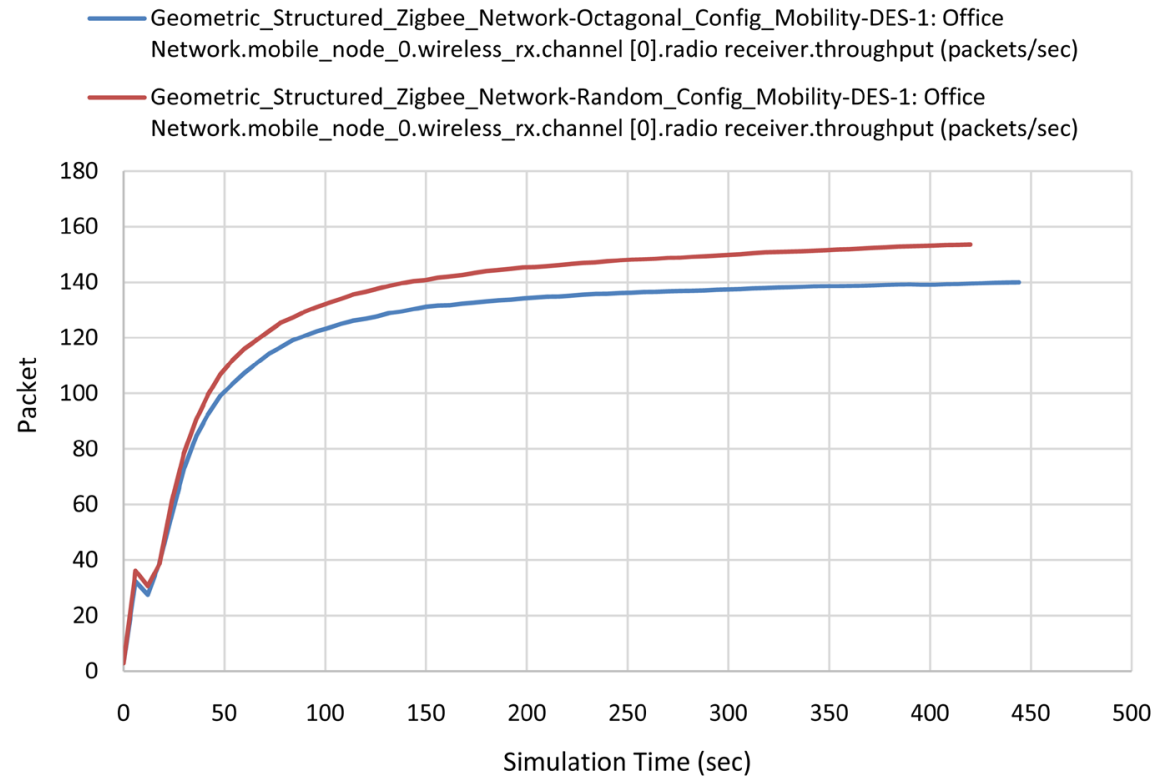

Figure 10. Average receiver throughput (packets/sec) for octagonal and random model. 


\section{Conclusions}

In this paper, mobility issue in ZigBee network has been analyzed based on Random and Octagonal mobility configuration model. The mobility models have adopted to make the ZigBee network more versatile and user friendly. The results are received from Riverbed based simulation. The simulation result demonstrates the comparative performance between two different schemes of sink mobility. According to the result, the network performance parameters of the mobile node's transmitter and receiver in the Octagonal configuration, such as queue size, queuing delay, bit error rate, packet loss ratio and signal to noise ratio show better result than the Random configuration. But the random configuration is better in throughput for both transmitter and receiver than the octagonal configuration. Nevertheless, the octagonal configuration shows comparatively better performance over the random configuration. The proposed ZigBee network runs with a great efficiency using the octagonal configuration mobility model. This model can be used in large scale ZigBee network formation.

In the future, the ZigBee network will be carried out in real life scenario with the Octagonal configuration mobility model. The two mobility models are analyzed in this article, but ZigBee mobile node's mobility with GPS tracking will be analyzed in the next.

\section{References}

[1] (2016) Specification, ZigBee. ZigBee Alliance. http://www.ZigBee.org

[2] Islam, N., Biddut, M.J.H., Swapna, A.I. and Jany, M.H.R. (2015) A Study on Priority Based ZigBee Network Performance Analysis with Tree Routing Method. Journal of Computer and Communications, 3, 1-10. http://dx.doi.org/10.4236/jcc.2015.38001

[3] Dhaka, H., Jain, A. and Verma, K. (2010) Impact of Coordinator Mobility on the Throughput in a ZigBee Mesh Networks. 2010 IEEE 2nd International Advance Computing Conference (IACC), Patiala, 19-20 February 2010, 279-284. http://dx.doi.org/10.1109/IADCC.2010.5422995

[4] Wu, Z., Chu, H., Pan, Y. and Yang, X. (2006) Bus Priority Control System Based on Wireless Sensor Network (WSN) and ZigBee. IEEE International Conference on Vehicular Electronics and Safety, Beijing, 13-15 December 2006, 148151. http://dx.doi.org/10.1109/icves.2006.371572

[5] Zhou, R., Zhao, C., Fu, L., Chen, A. and Ye, M. (2010) ZigBee-Based Vehicle Access Control System. Third International Symposium on Intelligent Information Technology and Security Informatics, Jinggangshan, 2-4 April 2010, 232235. http://dx.doi.org/10.1109/IITSI.2010.50

[6] Stevanovic, D. and Vlajic, N. (2008) Performance of IEEE 802.15.4 in Wireless Sensor Networks with a Mobile Sink Implementing Various Mobility Strategies. 33rd IEEE Conference on Local Computer Networks, Montreal, 14-17 October 2008, 680-688. http://dx.doi.org/10.1109/lcn.2008.4664265

[7] Dong, K. (2011) Performance and Fairness Enhancement in ZigBee Networks. Doctoral Dissertation, Delft University of Technology, TU Delft.

[8] Mouawad, A., Chalhoub, G., Habib, G. and Misson, M. (2013) A Performance Study of Mobile Nodes in a Wireless Sensor Network. 2013 Third International Conference on Communications and Information Technology (ICCIT), Beirut, 19-21 June 2013, 136-140. http://dx.doi.org/10.1109/ICCITechnology.2013.6579537

[9] Ddin, W.U. and Khan, B.M. (2014) Improved Quality of Service in Wireless Sensor Network with Mobile Sink. 2014 IEEE 17th International Multi-Topic Conference (INMIC), Karachi, 8-10 December 2014, 36-40. http://dx.doi.org/10.1109/inmic.2014.7096907

[10] Chaabane, C., Pegatoquet, A., Auguin, M. and Jemaa, M.B. (2012) An Efficient Mobility Management Approach for IEEE 802.15.4/ZigBee Nodes. 2012 IEEE 14th International Conference on High Performance Computing and Communication \& 2012 IEEE 9th International Conference on Embedded Software and Systems (HPCC-ICESS), 897-902.

[11] Mu, J. and Liu, K. (2010) Effect of Node Mobility and Network Dimension to the ZigBee Routing Method. 2010 6th International Conference on Wireless Communications Networking and Mobile Computing (WiCOM), Chengdu, 23-25 September 2010, 1-5. http://dx.doi.org/10.1109/wicom.2010.5601250

[12] Biddut, J.H., Islam, N., Jany, M.H.R. and Swapna, A.I. (2015) Performance Analysis of Large Scale ZigBee Network Design through Geometric Structure. 2015 2nd International Conference on Electrical Information and Communication Technology (EICT), Khulna, 10-12 December 2015, 263-268. http://dx.doi.org/10.1109/EICT.2015.7391958

[13] The Network Simulator-NS-2 (2016) http://www.isi.edu/nsnam/ns/

[14] The Network Simulator-NS-3 (2016) https://www.nsnam.org/

[15] (2016) OMNeT++: Discrete Event Simulator. https://omnetpp.org/

[16] Modeler, O.P.N.E.T. (2016) Riverbed Technology. Inc. http://www.riverbed.com 
[17] Shih, Y.Y., Chung, W.H., Hsiu, P.C. and Pang, A.C. (2013) A Mobility-Aware Node Deployment and Tree Construction Framework for ZigBee Wireless Networks. IEEE Transactions on Vehicular Technology, 62, 2763-2779. http://dx.doi.org/10.1109/TVT.2013.2245693

[18] Pacheco, R., Ordóñez, J. and Martínez, G. (2012) Energy Efficient Design of Building: A Review. Renewable and Sustainable Energy Reviews, 16, 3559-3573. http://dx.doi.org/10.1016/j.rser.2012.03.045

[19] Kim, T., Kim, S.H., Yang, J., Yoo, S.E. and Kim, D. (2014) Neighbor Table Based Shortcut Tree Routing in ZigBee Wireless Networks. IEEE Transactions on Parallel and Distributed Systems, 25, 706-716.

http://dx.doi.org/10.1109/TPDS.2014.9 